\title{
Lateral Hypothalamic Innervation of the Cerebral Cortex: Immunoreactive Staining for a Peptide Resembling but Immunochemically Distinct From Pituitary/Arcuate $\alpha$-Melanocyte Stimulating Hormone
}

\author{
CLIFFORD B. SAPER ${ }^{1}$ \\ Departments of Neurology and Anatomy and Neurobiology \\ Washington University School of Medicine, St. Louis, MO 63110 \\ and Departments of Pharmacological and Physiological Science and Neurology \\ University of Chicago, Chicago, IL 60637
}

HUDA AKIL AND STANLEY J. WATSON

Mental Health Research Institute, Department of Psychiatry

University of Michigan School of Medicine, Ann Arbor, MI 48109

Received 9 August 1985

\begin{abstract}
SAPER, C. B., H. AKIL AND S. J. WATSON. Lateral hypothalamic innervation of the cerebral cortex: Immunoreactive staining for a peptide resembling but immunochemically distinct from pituitary/arcuate a-melanocyte stimulating hormone. BRAIN RES BULL 16(1) 107-120, 1986.-The combination of retrograde transport of fluorescent dyes and indirect immunofluorescence has been used to study the putative neurotransmitter specificity of the tuberal lateral hypothalamic projection to the cerebral cortex. Injections of either fast blue or diamidino yellow dye into the cerebral cortex or hippocampus retrogradely labeled large, multipolar neurons scattered through the lateral hypothalamic area and zona incerta at the level of the ventromedial nucleus of the hypothalamus. Approximately $80 \%$ of these neurons stained immunohistochemically with an antiserum against $\alpha$-melanocyte stimulating hormone ( $\alpha$-MSH). A second population of smaller, predominantly bipolar $\alpha$-MSH-like immunoreactive neurons was seen in the arcuate nucleus and retrochiasmatic area, but none of these projected to the cerebral cortex. Immunohistochemical staining for ACTH (18-24), another proopiomelanocortin series peptide, or with an antiserum against $\alpha$-MSH (4-10) demonstrated only the second of these cell groups. Our results indicate that the tuberal lateral hypothalamic projection to the cerebral cortex contains a substance similar but not identical to $\alpha-\mathrm{MSH}$, and that this material is probably not derived from the same proopiomelanocortin precursor as true $\alpha$-MSH
\end{abstract}

Lateral hypothalamic area Cerebral cortex Alpha-melanocyte stimulating hormone $(\alpha-\mathrm{MSH})$ Adrenocorticotrophic hormone (ACTH)-proopiomelanocortin

SINCE the first demonstration of a hypothalamic projection to the cerebral cortex in the monkey by Kievet and Kuypers [1] in 1975, the existence of this pathway has been confirmed in a variety of mammalian species (see [19] for review). Only recently, however, has the organization of the hypothalamocortical projection been described in any detail
[19]. In the rat, the hypothalamus contains four discrete cell groups, each of which innervates the cerebral cortex in a different pattern. A cell group in the tuberal lateral hypothalamus (LHAt), consisting of neurons scattered through the lateral hypothalamic area and the zona incerta (at the level of the ventromedial nucleus of the hypothalamus), innervates

\footnotetext{
'Requests for reprints should be addressed to Dr. C. B. Saper, Department of Pharmacological and Physiological Sciences, University of Chicago, Pritzker School of Medicine, 947 East 58th Street, Chicago, IL 60637.
} 
the entire cortical mantle, including allocortical structures such as the hippocampus. These neurons tend to cluster in the perifornical region, the zona incerta and the area adjacent to the internal capsule. There is little apparent topographic order in their projection to the cerebral cortex, except in the perifornical region. Here, the neurons which project to the hippocampal formation tend to aggregate ventromedial to the fornix, those projecting to the cingulate cortex aggregate medial to the fornix, those sending axons to the sensory and motor areas cluster dorsal to the fornix and those projecting to lateral frontal cortex are mostly located lateral to the fornix.

Watson and Akil [27,28] and Guy and coworkers [3, 4, 7] have demonstrated a cell group in the LHAt of the rat which stains immunohistochemically with antisera against $\alpha$-melanocyte stimulating hormone $(\alpha-\mathrm{MSH})$. Unlike $\alpha$-MSH like immunoreactive (-lir) neurons in the arcuate nucleus of the hypothalamus, the LHAt neurons do not stain with antisera against other peptides derived from the proopiomelanocortin precursor. such as $\beta$-endorphin or the CLIP region of adrenocorticotrophic hormone (ACTH). To emphasize this biochemical difference in the lateral hypothalamic $\alpha$-MSH-lir neurons. Khachaturian and coworkers [9] termed them the "alpha-2" cell group.

The alpha-2 neurons are distributed in a manner similar to the cortical projection neurons in the tuberal lateral hypothalamus. Several groups of investigators have demonstrated widespread $\alpha$-MSH-lir innervation of the cerebral cortex, which they have attributed to fibers arising from the alpha-2 group $[4,8,9,12,13,22]$. Recently Shiosaka and coworkers and Kohler and colleagues have used combined retrograde tracer and immunohistochemical methods to confirm that alpha-2 neurons do indeed innervate the hippocampal, infralimbic, entorhinal and parietal cortical areas.

We have used similar methods to investigate this projection system in greater detail, confirming the earlier observations and extending them to several additional cortical areas. Furthermore, we have used two new antisera, prepared against peptide fragments of $\alpha-\mathrm{MSH}$ and ACTH, as biochemical probes to help determine whether the innervation of the cerebral cortex by the alpha- 2 group contains genuine $\alpha-\mathrm{MSH}$.

\section{METHOD}

Under chloral hydrate anesthesia, injections of $50 \mathrm{nl}$ of $5 \%$ solutions of diamidino yellow and fast blue dyes were placed stereotaxically into different pairs of cortical areas in a series of six adult male Sprague Dawley rats (see Table 1) using a micropipette syringe, as previously described [19]. The injection sites, including hippocampus, somatic sensory, motor, infralimbic/prelimbic and insular areas, were chosen on the basis of an earlier study on the distribution of hypothalamo-cortical projections [19] to reflect the organization of this system vis-a-vis allocortex, periallocortex and
TABLE I

INJECTION SITES OF FLUORESCENT DYES IN CEREBRAL CURTEX

\begin{tabular}{lll}
\hline Experiment & $\begin{array}{l}\text { Fast Blue } \\
\text { Injection }\end{array}$ & $\begin{array}{l}\text { Diamidino Yellow } \\
\text { Injection }\end{array}$ \\
\hline RF 101 & $\begin{array}{l}\text { prelimbic. } \\
\text { infralimbic }\end{array}$ & insular \\
RF 102 & insular & somatic sensory \\
RF 116 & hippocampus & prelimbic \\
RF 120 & somatic sensory & motor \\
RF 137 & motor & somatic sensory \\
RF 140 & somatic sensory & motor
\end{tabular}

isocortex. Following one to two weeks survival, the animals received an intraventricular injection of $150 \mu \mathrm{g}$ colchicine in $10 \mu \mathrm{l}$ of $0.9 \%$ saline. Forty hours later, the animals were perfused with $0.9 \%$ saline, followed by $4 \%$ paraformaldehyde in $0.1 \mathrm{M}$ phosphate buffer, $200 \mathrm{ml}$ at $\mathrm{pH} 6.5$, then $200 \mathrm{ml}$ at $\mathrm{pH} \mathrm{8.5}$. The brains were removed and $50 \mathrm{~km}$ sections of the injection sites and hypothalamus were cut on a freezing microtome. Sections through the injection sites were mounted on glass slides and viewed, without coverslips, using a Leitz Ortholux microscope with Ploem fluorescence illumination. filter A, to verify injection placement.

Sections through the hypothalamus were stained immunohistochemically for $\alpha$-MSH by the indirect immunofluorescence method. Briefly, sections were first incubated in $0.1 \mathrm{M}$ phosphate buffered $0.9 \%$ saline, $\mathrm{pH} \mathrm{7.4,} \mathrm{(PBS)} \mathrm{con-}$ taining $0.25 \%$ Triton $X-100$ for one hour at room temperature. They were then washed in PBS and incubated for another hour in PBS containing $3 \%$ normal goat serum (PBS-G) as a blocking step to inhibit nonspecific binding in later antisera incubations. Following another wash in PBS-G, the sections were incubated overnight at $4^{\circ} \mathrm{C}$ in PBS-G containing a 1:1000 dilution of $\alpha$-MSH antiserum. The specificity of this antiserum has been described elsewhere $[8]$. The next day, the sections were washed again in PBS-G and incubated with fluorescein isothiocyanate (FITC)-labeled goat-antirabbit IgG antiserum (Miles), diluted 1:50 in PBS-G, for one hour at room temperature. The sections were then washed in PBS, mounted on gelatin-coated glass slides and coverslipped with glycerin. The sections were viewed, as noted above, using the $A$ filter system for fast blue and diamidino yellow, and the 12 filter system for FITC.

Immunohistochemical control sections through the hypothalamus were treated in exactly the same manner as out: lined above, except that the primary antiserum was adsorbed with $50 \mu \mathrm{g}$ of synthetic $\alpha$-MSH (Sigma) per ml diluted antiserum. Some hypothalamic sections were alternatively stained with an antiserum against the ACTH 18-24 amino

\section{FACING PAGE}

FIG. 1. A pair of photomicrographs of sections through the most rostral part of the tuberal lateral hypothalamus (LHAt), at the level of the caudal part of the paraventricular nucleus to illustrate the distribution of the $\alpha$-MSH-like immunoreactive (-lir) neurons in the hypothatamus of the rat. In each figure, panel A shows a $50 \mu \mathrm{m}$ frozen section stained immunohistochemically for $\alpha$-MSH, while panel B shows a thioninstained $15 \mu \mathrm{m}$ paraffin-embedded section through the comparable region in another brain, to demonstrate the cytoarchitecture of the area. Note that the medial part of the paraventricular nucleus contains an $\alpha$-MSH-lir terminal field, but that the immunoreactive neurons are located along the borders of the nucleus. A distinct population of immunoreactive neurons in the arcuate nucleus is also visible. Scale for Figs. $1-5=250 \mu \mathrm{m}$ (the slight shrinkage of the paraffin-embedded brain accounts for the apparent difference in scale between panels $A$ and $B$ ). 




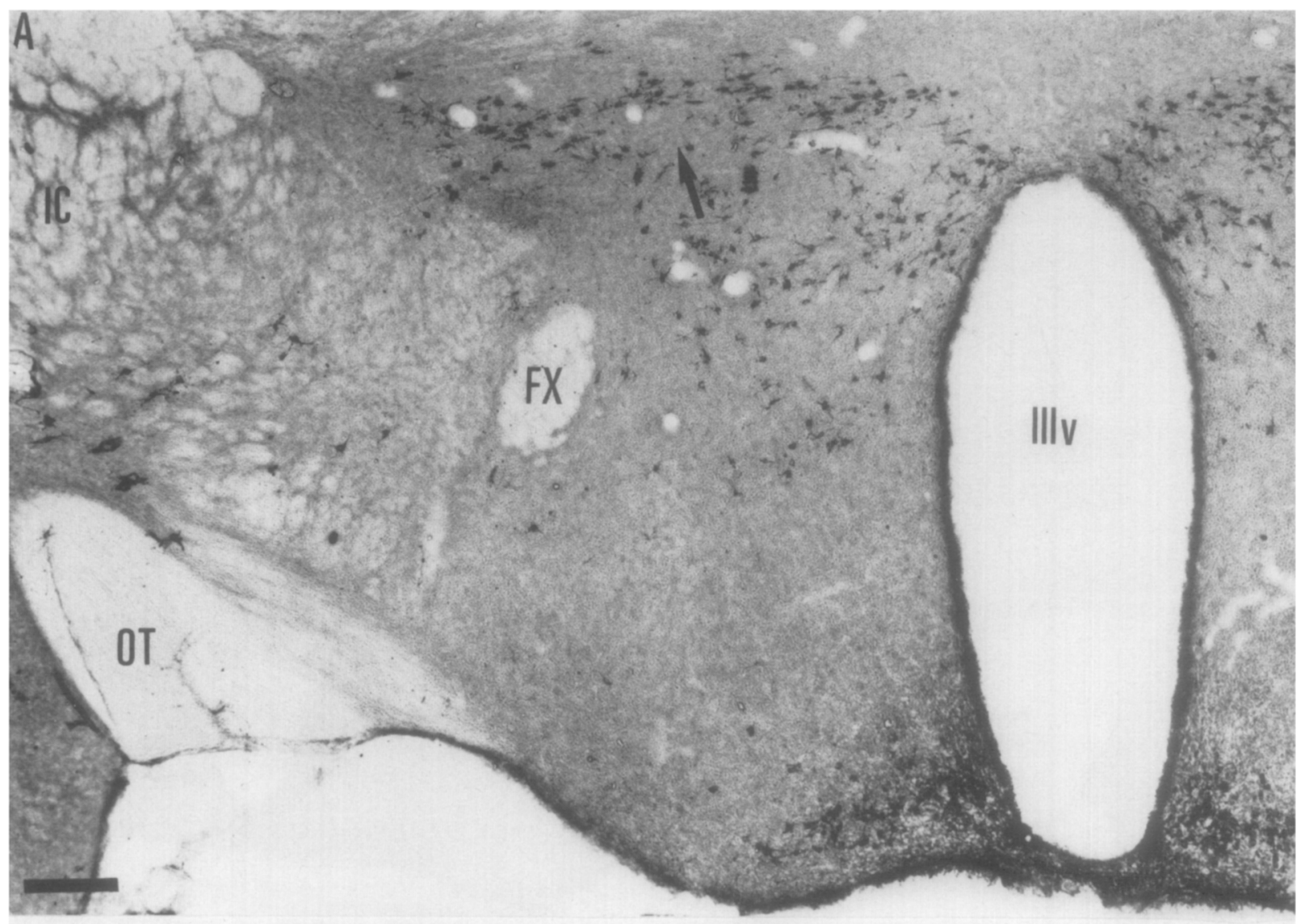

P.

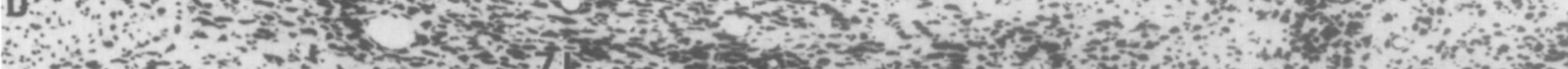

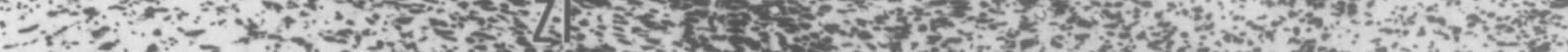

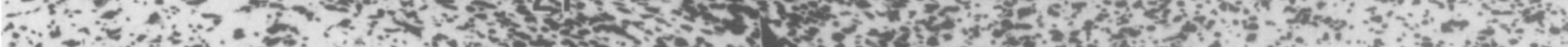

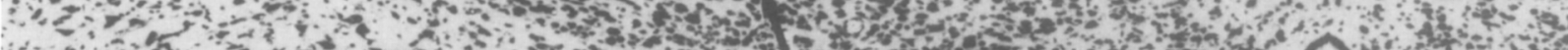
a 7 and 5-3,

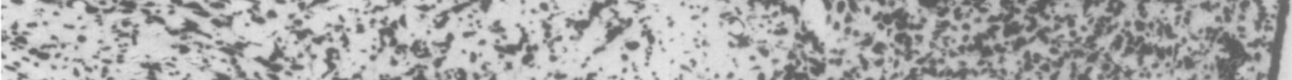

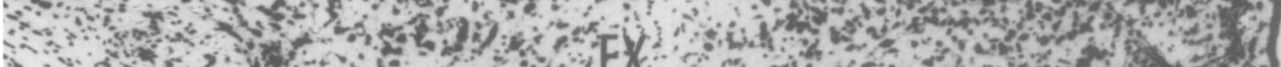
s.

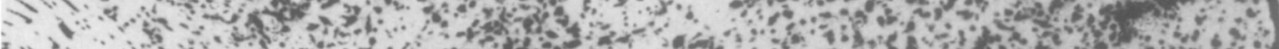
1) a jo

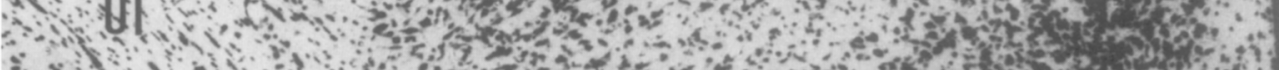
80 a

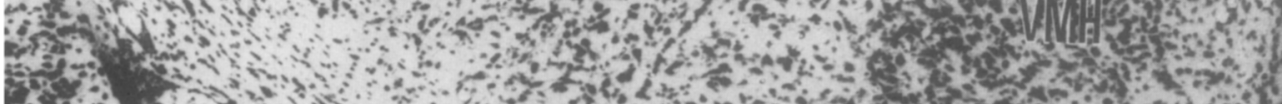

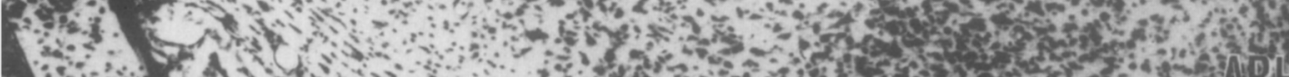
13.

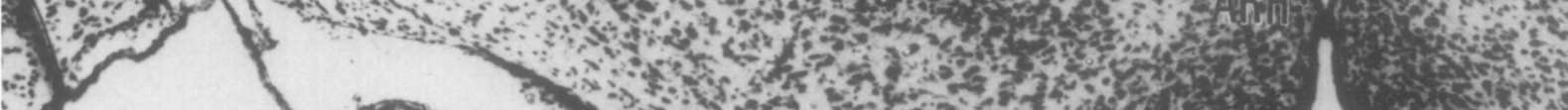
(2) 10 (1) 
acid fragment, using the same primary antiserum as Khachaturian et al. [8], diluted 1:1000, in the above protocol.

In four additional rats, the staining of the hypothalamus with the $\alpha$-MSH antiserum was compared to that obtained using the antiserum against ACTH (18-24), or an antiserum against the 4-10 region of $\alpha$-MSH (Watson et al., in preparation). These animals received intraventricular colchicine, and three were processed as outlined above, except that the goat anti-rabbit antiserum was labeled with horseradish peroxidase instead of FITC. The sections were then stained with $0.01 \% \mathrm{H}_{2} \mathrm{O}_{2}$, and $0.05 \%$ diaminobenzidine in phosphate buffer before being mounted on glass microscope slides, dehydrated and coverslipped with Histoclad. These permanent sections allowed more detailed observations of the $\alpha$ MSH-lir, which fades rapidly in fluorescent material. Ten micron sections from the hypothalamus of the fourth animal were cut on a cryostat, and the sections were stained on the slide using the immunoperoxidase method, with antisera against $\alpha$-MSH or $\alpha$-MSH (4-10). As controls, sections were also stained with the $\alpha$-MSH(4-10) antiserum after adsorption with $\alpha$-MSH (4-10), $\alpha$-MSH (1-7) or ACTH (11-19) peptides.

\section{RESULTS}

Immunohistochemically labeled neurons were found in two distinct groups (see Figs. 1-5). One cluster of very heavily stained $\alpha$-MSH-lir neurons was seen in the arcuate nucleus and adjacent retrochiasmatic area. These were 15-18 $\mu \mathrm{m}$ in diameter and mostly bipolar in shape. The second $\alpha$-MSH-lir cell group was located in the lateral hypothalamus and adjacent areas, at the level of the ventromedial nucleus. These 20-25 $\mu \mathrm{m}$ multipolar neurons stained slightly less heavily than those in the arcuate nucleus, but were approximately twice as numerous.

The $\alpha$-MSH-lir neurons in the lateral hypothalamus were coextensive with the LHAt cortical-projections cell group (see [19]) and distributed in an identical pattern. Rostrally, these neurons were first seen along the margins of the posterior part of the paraventricular nucleus, and the adjacent posterior cell cluster of the anterior hypothalamic area (see Fig. 1). More caudally the $\alpha$-MSH-lir neurons invaded the zona incerta and lateral and dorsal hypothalamic areas, and were found along the borders of the ventromedial and dorsomedial nuclei (Figs. 2-3). In the LHAt, $\alpha$-MSH-lir perikarya tended to cluster in the perifornical region and along the ventral margin of the zona incerta and the medial aspect of the internal capsule. The aggregations of large neurons in these areas often corresponded to cell clusters which were apparent in Nissl-stained sections at the same level (Figs. 3-5). Based upon these observations it appears that a substantial proportion of all the large cells in these parts of the lateral hypothalamus stain with antiserum against $\alpha$-MSH.

In sections stained using the antisera against ACTH (1824) or $\alpha$-MSH (4-10), the only immunoreactive neurons in the hypothalamus were found in the arcuate nucleus and adjacent retrochiasmatic area (see Fig. 6). These neurons were cytologically identical to, and were distributed in the same manner as the arcuate area $\alpha$-MSH-lir perikarya. Adsorption of the $\alpha$-MSH or the $\alpha$-MSH (4-10) antisera with $\alpha$-MSH peptide abolished all staining in the hypothalamus. Staining was also blocked by adsorbing the $\alpha$-MSH $(4-10)$ antiserum with $\alpha$-MSH (4-10) peptide, but not with $\alpha$-MSH (1-7) or ACTH (11-19) peptides.

The pattern of retrogradely labeled hypothalamic neurons following injections of either fluorescent dye into the motor, somatic sensory, prelimbic/infralimbic, insular or hippocampal cortex was identical with that which we have previously reported [19]. Retrogradely labeled neurons were found scattered through the LHAt, but were most densely localized in the perifornical region, the zona incerta and along the medial border of the internal capsule (see Fig. 7). Occasional labeled neurons were found in an identical distribution in the contralateral hypothalamus.

In each experiment, from 74 to $91 \%$ of the LHAt neurons retrogradely labeled with each dye stained immunohistochemically for $\alpha$-MSH (see Figs. 7,8). There was no apparent relationship of the percentage of double-labeled neurons with the cortical area injected. Most of the retrogradely labeled neurons which did not stain for $\alpha$-MSH were located along the ventral margins of the LHAt cortical projection cell group. In each experiment, only occasional LHAt cells were retrogradely labeled with both fluorescent dyes; virtually all of these retrogradely double-labeled neurons were $\alpha$ MSH-lir. Similarly, while only a few retrogradely labeled neurons were seen in the contralateral LHAt, nearly all of these stained for $\alpha$-MSH (Fig. 8).

\section{DISCUSSION}

Our findings provide direct evidence that $\alpha$-MSH-lir neurons in LHAt are the source of a major projection to the entire cortical mantle. This possibility was first raised by the observation that neurons in the LHAt and fibers in the cerebral cortex both stained with antisera against $\alpha$-MSH, but not other proopiomelanocortin peptides $[4,8,9,13,22]$. Khachaturian and colleagues [8] have traced $\alpha$-MSH-lir fibers from the hypothalamus to the cerebral cortex through three main pathways: (1) some $\alpha$-MSH-lir fibers enter the fornix, and follow that pathway back to the hippocampal formation; (2) a medial contingent of $\alpha$-MSH-lir fibers run rostrally in the medial forebrain bundle, over the genu of the corpus callosum, and into the medial prefrontal cortex and the cingulate bundle; and (3) a lateral group of $\alpha$-MSH-lir fibers enters the ansa peduncularis, and runs into the external capsule. These pathways are identical to those which we have recently shown are taken by posterior lateral hypothalamic fibers which innervate the cerebral cortex [19].

The magnitude of the $\alpha$-MSH-lir projection to the cerebral cortex is striking. In our previous retrograde transport study of this pathway, it was estimated that the number of LHAt neurons projecting to the cerebral cortex was approximately $53 \%$ of the total for the magnocellular basal nucleus. As up to $90 \%$ of the LHAt cortical projection neurons appear to be $\alpha$-MSH-lir, this population is at least $50 \%$ of the size of the cholinergic cortical projection, and probably greater (since

\section{FACING PAGE}

FIG. 2. A pair of sections at the level of the rostral part of the ventromedial nucleus. The immunoreactive neurons outline the caudal extreme of the paraventricular nucleus (arrow) and the ventromedial nucleus, but invade the zona incerta and caudal part of the anterior hypothalamic area. 
not all magnocellular basal neurons stain for choline acetyltransferase-see [18]). It is difficult to appreciate the magnitude of the population of LHAt neurons which project to cerebral cortex in retrograde transport studies, as the labeled neurons are usually scattered across a large area. with minimal topographic clustering. However, in sections stained immunohistochemically for $\alpha-\mathrm{MSH}$, a substantial proportion of all the large neurons in the lateral hypothalamus contained immunoreactive material (see Figs. 1-5). The proportion of the $\alpha$-MSH-lir neurons in the LHAt which innervate the cerebral cortex can be estimated from our experiments. We typically retrogradely labeled as many as 5-10\% of the $\alpha$-MSH-lir neurons in the LHAt from a single injection site. Considering the small size of the injections which we used, and the paucity of axonal branching which we observed, a very large percentage of $\alpha$-MSH-lir neurons in the LHAt must innervate the cerebral cortex. Therefore, the impressive extent of the immunohistochemically stained cell group may, perhaps, serve as a rough indicator of the magnitude of the $\alpha$-MSH-lir projection from the LHAt to the cerebral cortex.

Kohler and Swanson [13] used techniques similar to those employed in this study to show that most of the neurons in LHAt which innervate the hippocampal formation and the infralimbic and entorhinal cortical areas are $\alpha$-MSH-lir. Shiosaka and coworkers [22] made very large injections of horseradish peroxidase-biotin conjugate into the "parietal cortex" in rats and showed that retrogradely labeled neurons in the zona incerta stained immunohistochemically for $\alpha$-MSH. The results of the present study, combined with our earlier, more extensive retrograde transport study [19], confirm and extend these observations. The $\alpha$-MSH immunoreactive neurons distributed through the perifornical region, the zona incerta and the lateral hypothalamic area represent the origin of a system of projections which appears to innervate the entire cortical mantle from the hippocampus through the motor and sensory isocortex. This projection is similar to that from the other hypothalamic cell groups, in the posterior lateral hypothalamic area and the tuberomammillary nucleus, each of which innervates the entire cerebral cortex, including the hippocampal formation. The cortical projection neurons in the tuberomammillary nucleus have recently been reported to stain immunohistochemically for glutamic acid decarboxylase, adenosine deaminase and histamine $[14,15,25]$. A few posterior lateral hypothalamic cortical projection neurons stain immunohistochemically for cholecystokinin $[5,19]$ but for the most part the neurotransmitter for this cell group remains unexplored.

Despite the striking immunohistochemical staining of the LHAt cortical projection with antisera against $\alpha$-MSH, the definitive identification of the neuropeptide contained in these neurons remains to be resolved. We have confirmed the earlier observation that the LHAt neurons do not stain for other proopiomelanocortin series peptides, such as ACTH or $\beta$-endorphin $[3,4,8,26-28]$, indicating that the $\alpha$-MSH-lir substance probably arises by a different biosynthetic route than pituitary or arcuate nucleus $\alpha-\mathrm{MSH}$. In addition, an antiserum against the 4-10 region of $\alpha$-MSH stained the arcuate, but not lateral hypothalamic. $\alpha$-MSH-lir neurons.

These results suggest that the $\alpha$-MSH-lir material in lateral hypothalamic neurons differs from that in arcuate neurons by some determinant in the $\alpha$-MSH 4-10 region. This could reflect a difference in amino acid sequence, or merely a conformational change in the molecule in situ. The former possibility was suggested by Jegou and colleagues [7] who reported that $\alpha$-MSH-lir material from the dorsolateral hypothalamus co-chromatographed with synthetic $\alpha$-MSH and possessed similar bioactivity. However. it is not clear whether the dorsolateral hypothalamic $\alpha$-MSH-lir was derived from "alpha-2" cell bodies or from the axons of arcuate nucleus neurons. To make this distinction, it will probably be necessary to develop cDNA probes for in situ hybridization, in order to localize mRNA sequences for proopiomelanocortin and related molecules to individual hypothalamic neurons.

Finally, a number of behavioral studies have examined the effects of intracerebroventricular administration of $\alpha$-MSH on attentional, arousal or motivational mechanisms $[11,24]$. The behavioral effects of $\alpha$-MSH may be due to its activity at subcortical sites. Altcrnatively. it may act at cortical receptors for the "alpha-2" peptide [11], which may serve as a neurotransmitter or neuromodulator in hypothalamic regulation of various behaviors at a cortical level

The diffuse distribution of the LHAt projection to the cerebral cortex suggests that it may play a role in regulating the overall state of cortical arousal. However, most neurons which contribute to this pathway appear to innervate restricted terminal areas, roughly confined to a single cytoarchitectonic field [19]. This system may therefore be capable of selectively mediating many of the consummatory, thermoregulatory or sexual behaviors which can be elicited by experimental manipulation of the hypothalamus $[1,2,6,16,17$, $20,21,23,29$ ! .

FIGS. 3, 4 AND 5

FOLLOWING PAGES

FIG. 3. A pair of sections at the mid-ventromedial nucleus level. Clusters of immunoreactive neurons adjacent to the internal capsule (arrow) and dorsolateral to the fornix (arrowhead) can be seen to correspond to groups of large Nissl-stained neurons in LHAt. A few immunoreactive neurons are seen along the lateral border of the ventromedial nucleus, but these are distinct from the smaller $\alpha$-MSH-lir neurons in the arcuate nucleus.

FIG. 4. A pair of sections through the caudal part of the ventromedial nucleus. Note that clusters of neurons corresponding to the $\alpha$-MSH-lir perikarya adjacent to the internal capsule (arrow) and the fornix (arrowhead) are easily distinguished in the comparable Nissl-stained section. An $\alpha$-MSH-lir terminal field is apparent in the pars compacta of the dorsomedial nucleus (curved arrow). Heavy $\alpha$-MSH-lir staining is also seen in the dorsal part of the external lamina of the median eminence.

FIG. 5. A pair of sections at the most caudal level of LHAt. The clusters of $\alpha$-MSH-lir neurons along the edge of the internal capsule (arrows) and surrounding the fornix (arrowheads) can be seen to correspond to groups of large Nissl-stained neurons. 


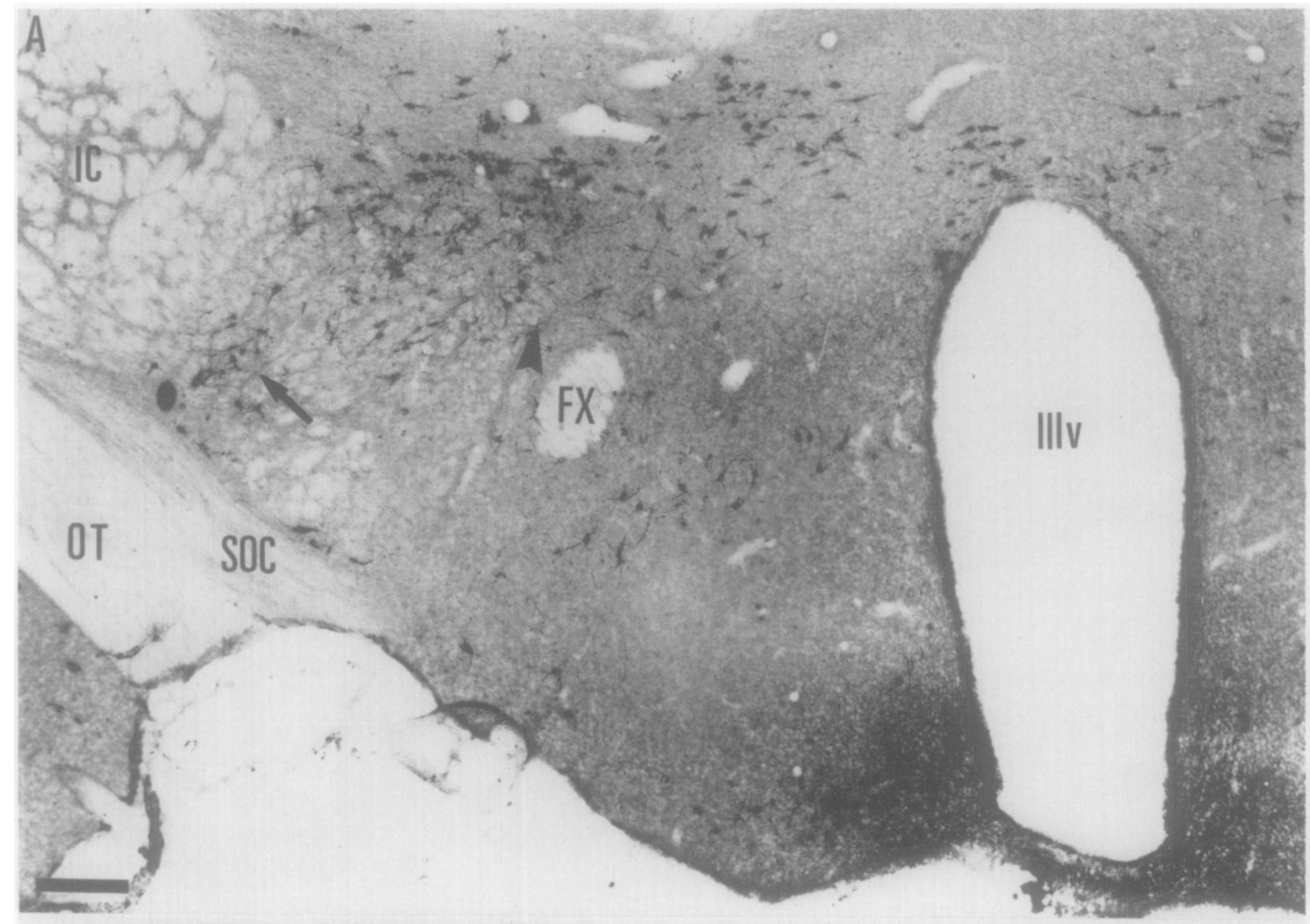

1.



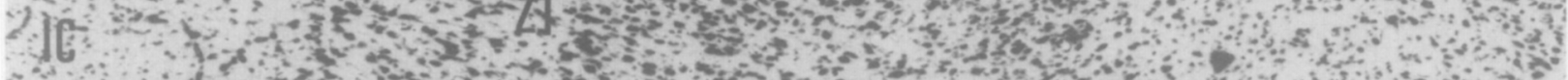



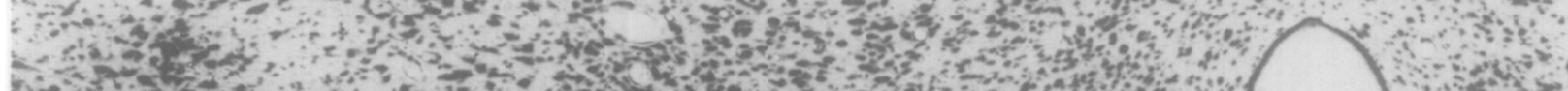

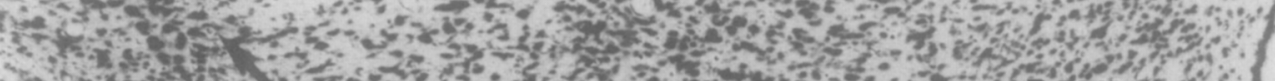

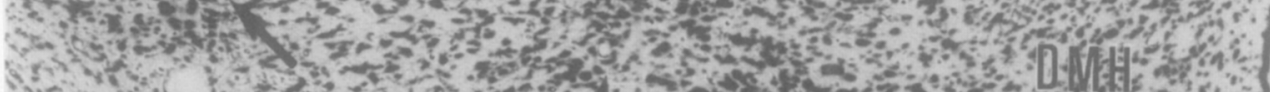

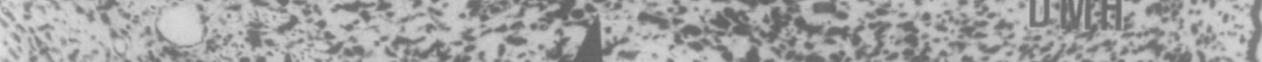

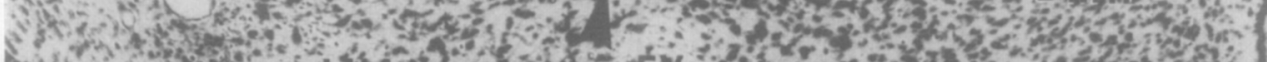
s.t.



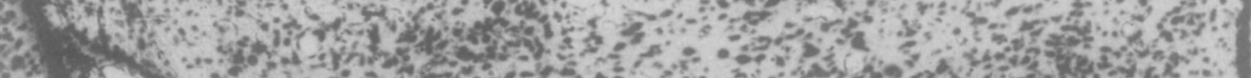

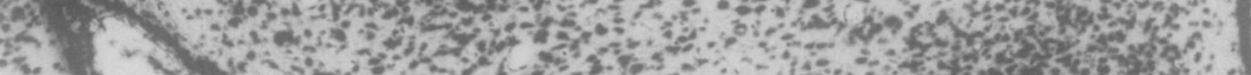
(3)

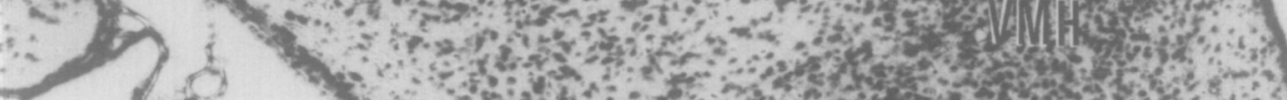

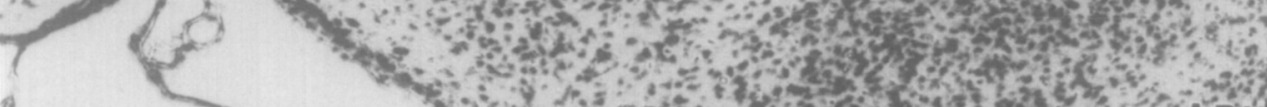

A

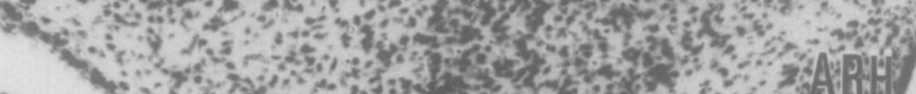

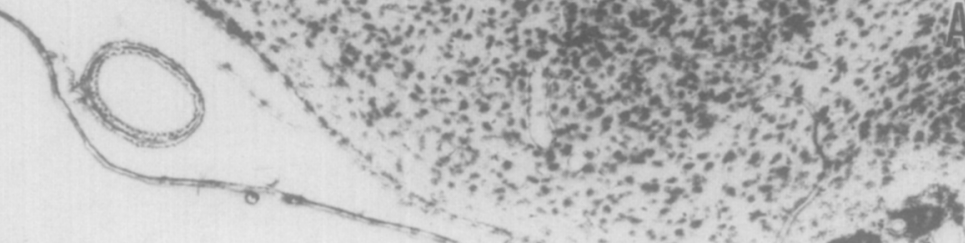

FIG. 3. 

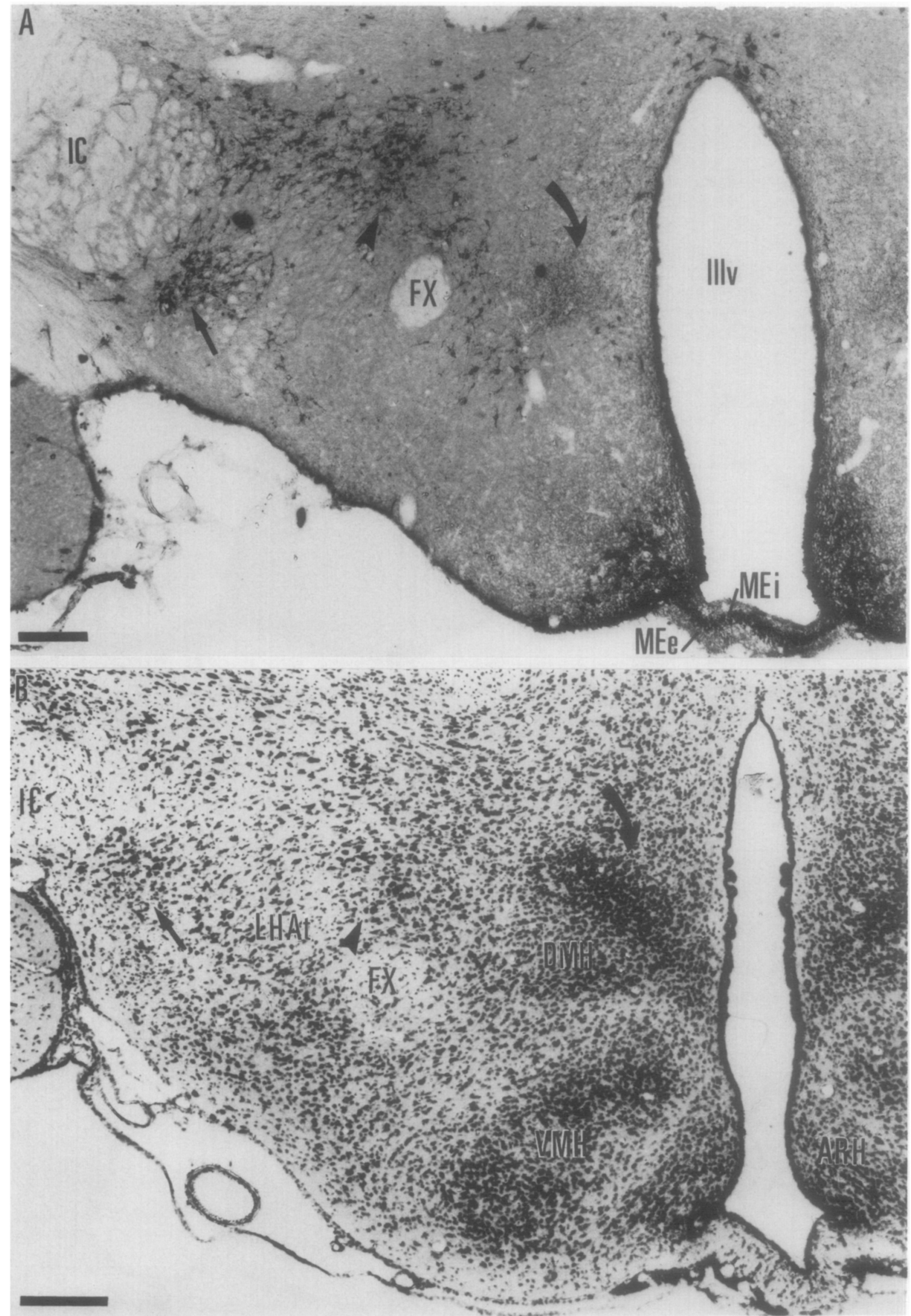

FIG. 4. 


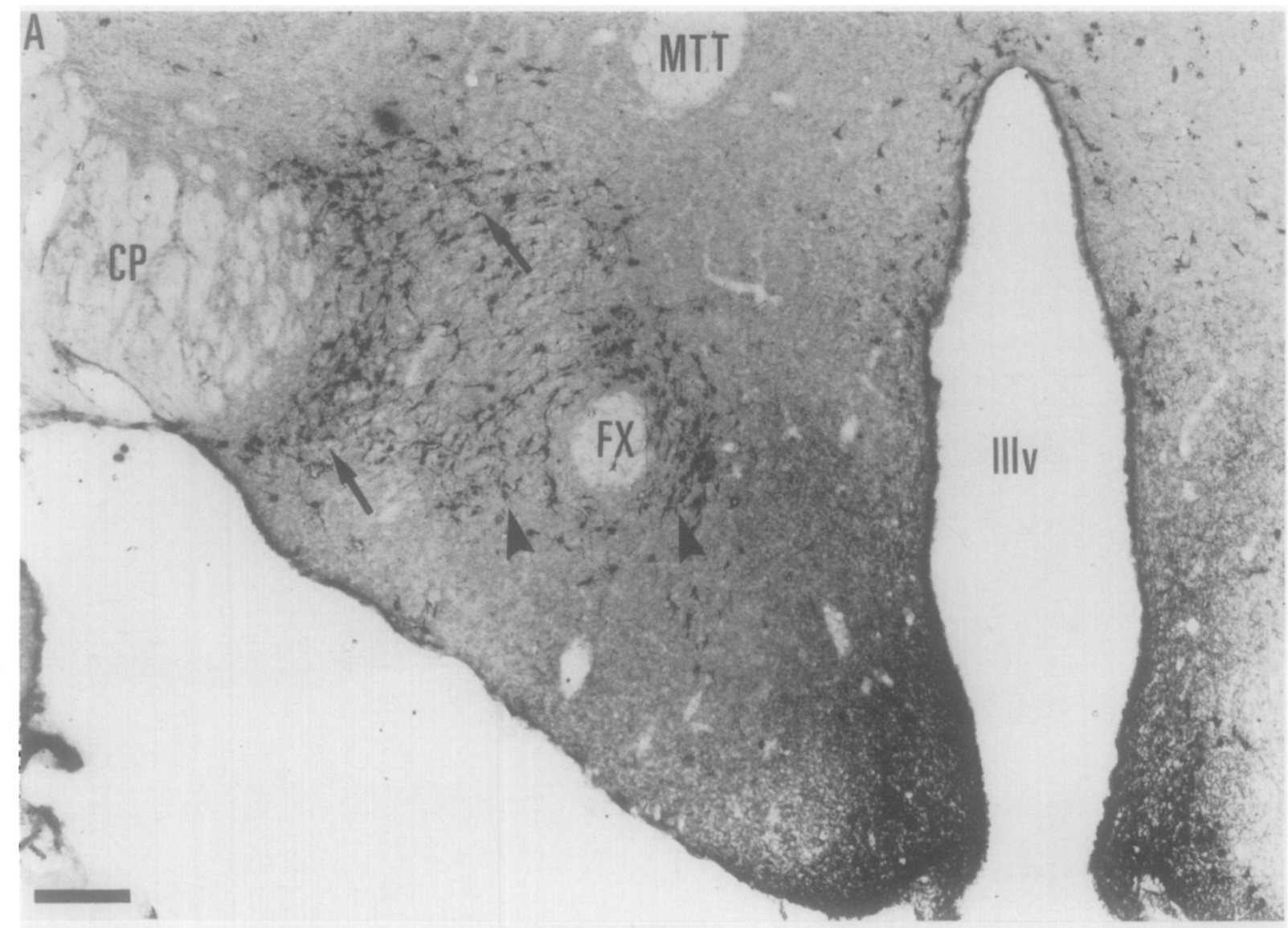
(19)

FIG. 5. 

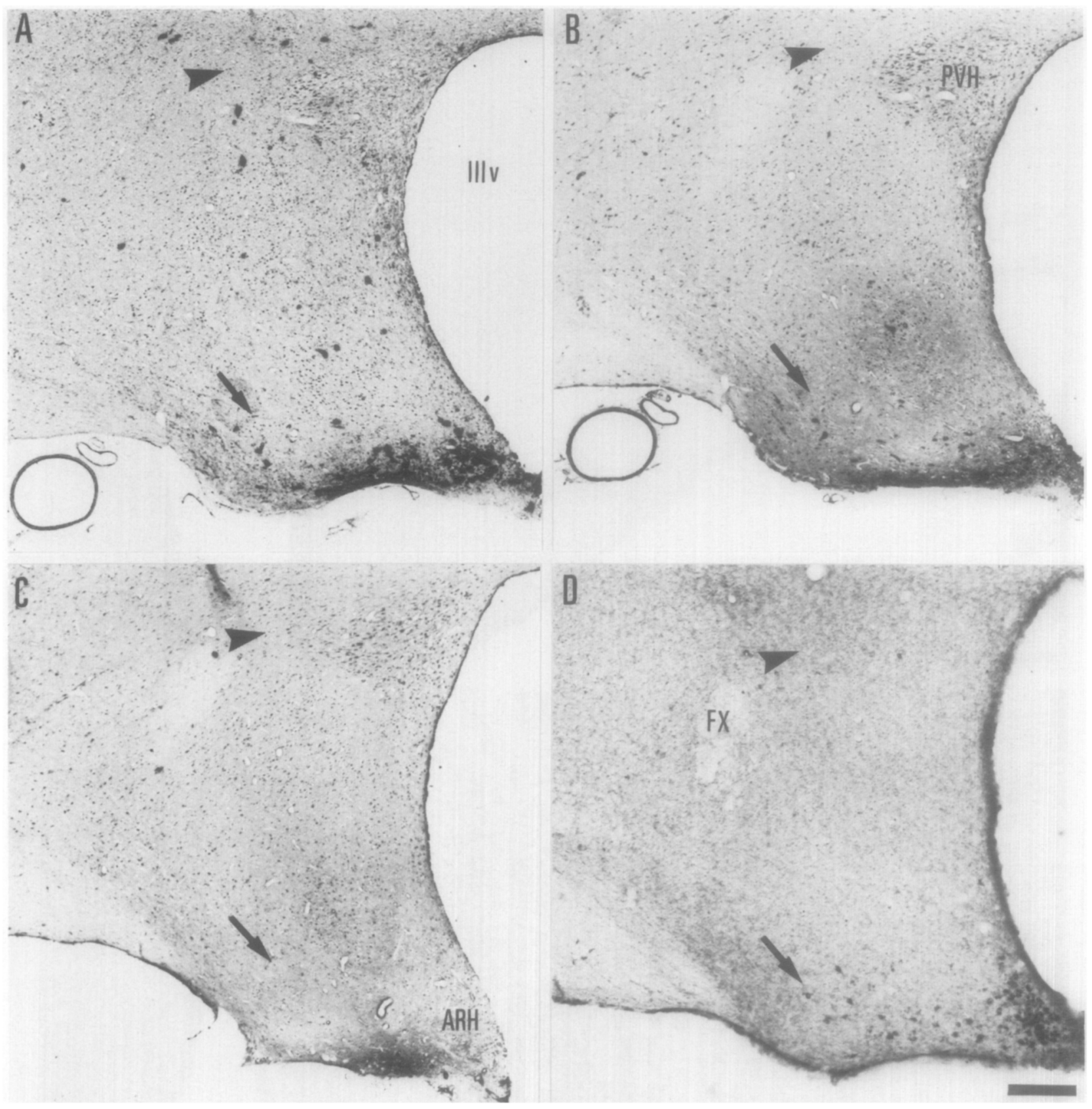

FIG. 6. A series of photomicrographs of sections through the hypothalamus at about the same level as shown in Fig. I. to illustrate the differential staining of the two $\alpha$-MSH-lir cell groups with different antisera. The section shown in panel A wits stained with an antiserum prepared against $\alpha$-MSH. In this $10 \mu \mathrm{m}$ thick section, as well as those shown in panels $B$ and $C$, nuclei of neurons and glia have picked up light, non-specific staining; only the neurons with very dark cytoplasmic staining are immunohistochemically labeled. The arrowhead indicates a group of stained neurons along the lateral margin of the paraventricular nucleus. The arrow points to a collection of stained neurons in the arcuate nucleus and retrochiasmatic area. An adjacent section stained with an antiserum against the $\alpha$-MSH 4-10 region is shown in panel B. Note that only the arcuate-retrochiasmatic neurons are stained by this antiserum. Adsorption of the $\alpha$-MSH (4-10) antiserum with $\alpha$-MSH (4-10) peptide completely blocked all staining (panel C). Staining a similar $50 \mu \mathrm{m}$ seetion with an antiserum against ACTH (18-24) (panel D) demonstrates staining only of the arcuate-retrochiasmatic cell group, similar to thit seen with the $\alpha$-MSH (4-10) antiserum. Scale $=250 \mu \mathrm{m}$. 
A

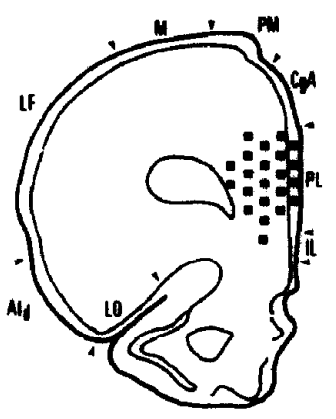

B

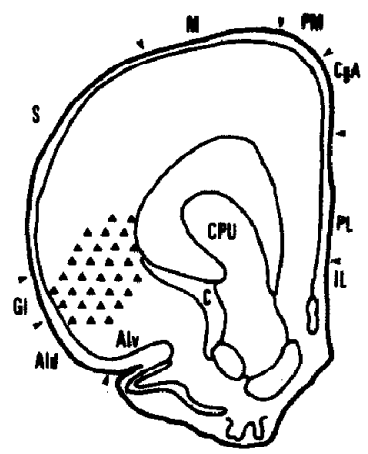

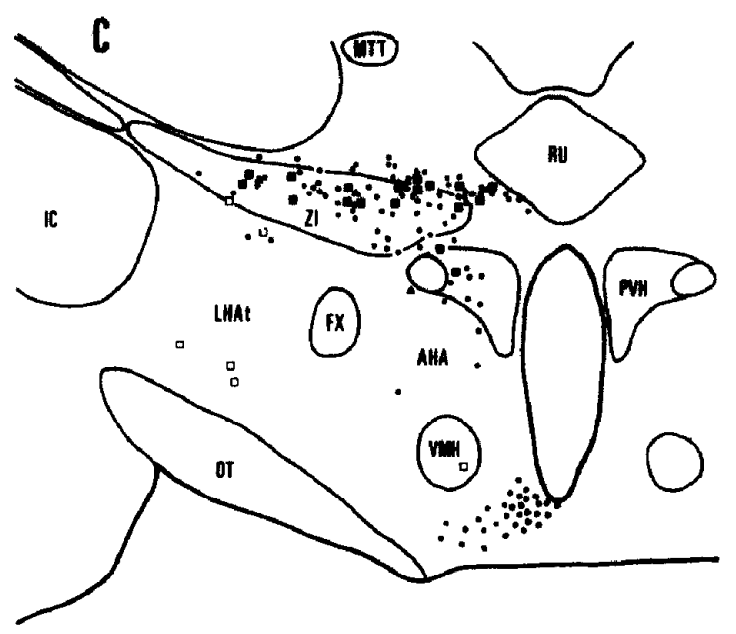
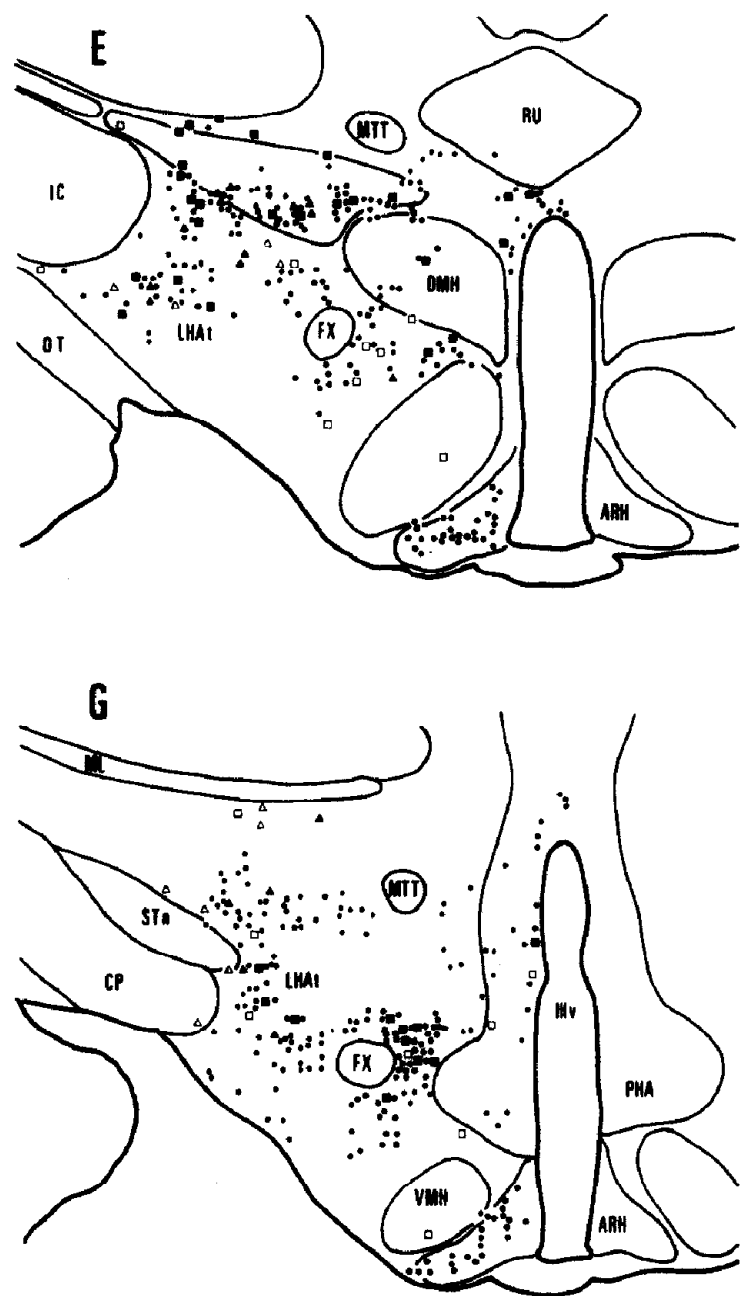

FIG. 7. A series of line drawings to illustrate the distribution of retrogradely labeled and $\alpha$-MSH-lir neurons in the hypothalamus in experiment RF 101, following injections of fast blue dye into the infralimbic cortex (filled squares in panel $A$ ) and diamidino yellow dye into the insular cortex (filled triangles in panel B). Labeled neurons are shown at five levels of the hypothalamus, corresponding to those shown in photomicrographs in Figs. 1-5, $\alpha$-MSH-lir neurons which were retrogradely labeled with fast blue dye (from the infralimbic cortex) are illustrated by filled squares; $\alpha$-MSH-lir neurons which were retrogradely labeled with diamidino yellow dye (from the insular cortex) are shown as filled triangles. Retrogradely labeled neurons which were not $\alpha$-MSH-lir are illustrated by the corresponding open symbols. $\alpha$-MSH-lir neurons which were not retrogradely labeled are shown as small dots. Note that, in this experiment, the retrogradely labeled $\alpha$-MSH-lir neurons were distributed throughout the $\alpha$-MSH-lir cell population. Retrogradely labeled neurons which were not $\alpha$-MSH-lir tended to be more ventrally located. No neurons containing both retrograde labels were seen in the illustrated sections. 

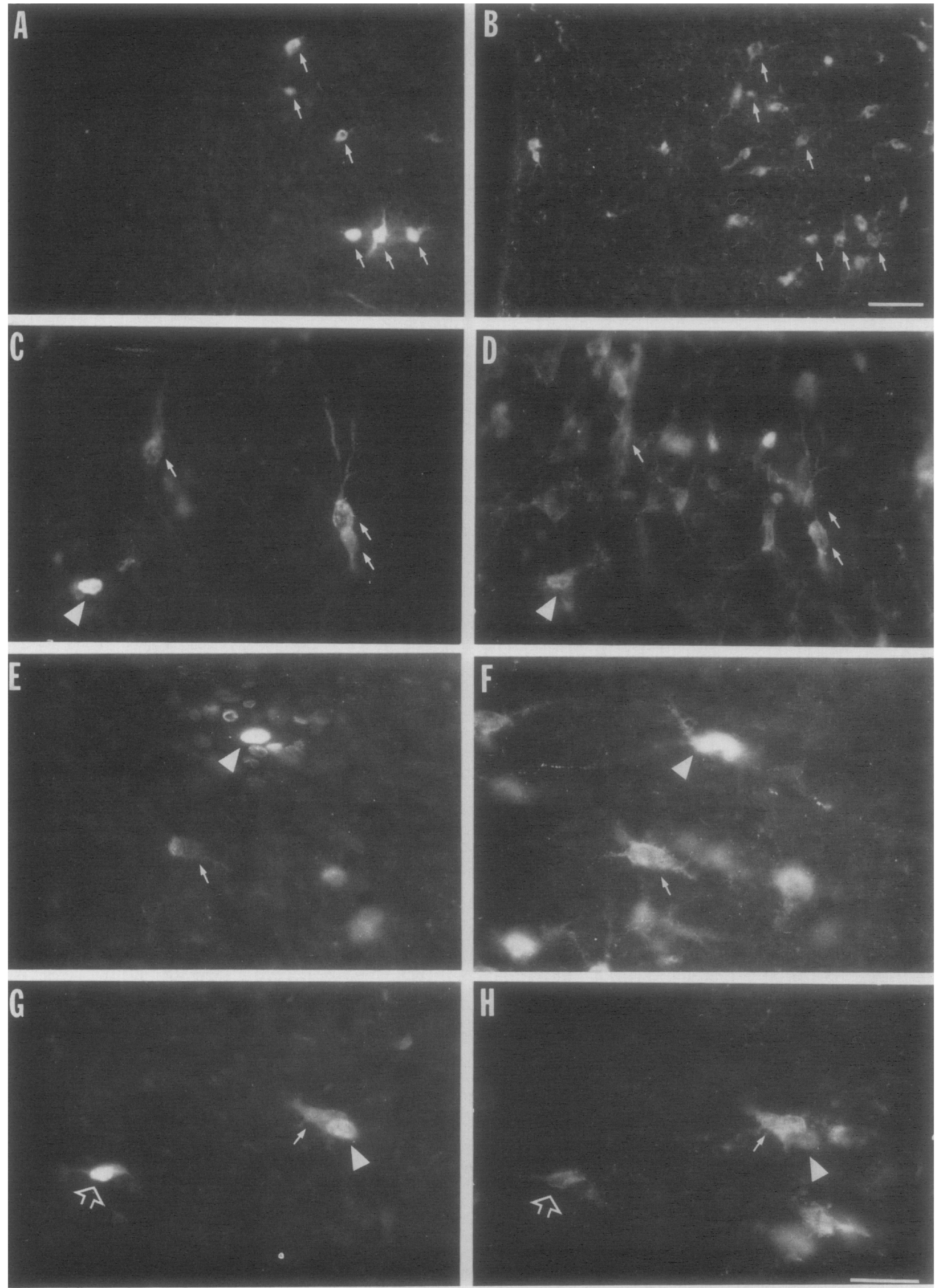


\section{ACKNOWLEDGEMENTS}

The authors thank Dr. Henry Khachaturian for helpful discussion and critical review of the manuscript, Ms. Quan Hue Ha for technical assistance and Mrs. Patti Nacci for help preparing the manuscript. This work was supported by USPHS grant NS18669 (C.B.S.) and NIDA grants DA02265 and DA00254 (S.J.W. and H.A.). C.B.S. is the recipient of NINCDS Teacher-Investigator Development Award NS00631 and a McKnight Scholar Award, and S.J.W. received NIDA award DA01165.

\section{REFERENCES}

1. Boulant, J. A. Hypothalamic control of thermoregulation. Neurophysiological basis. In: Behavioral Studies of the Hypothalamus. Handbook of the Hypothalamus, vol 3, part A, edited by P. J. Morgane and J. Panksepp. New York: Marcel Dekker, 1980 , pp. 1-82.

2. Grossman, S. P., D. Dacey, A. E. Halaris, T. Collier and A. Routtenberg. Aphagia and adipsia after preferential destruction of nerve cell bodies in hypothalamus. Science 202: 537-539, 1978.

3. Guy, J., R. Leclerc, H. Vaudry and G. Pelletier. Identification of a second category of $\alpha$-melanocyte-stimulating hormone $(\alpha-\mathrm{MSH})$ neurons in the rat hypothalamus. Brain Res 199: 135$146,1980$.

4. Guy, J., H. Vaudry and G. Pelletier. Differential projections of two immunoreactive $\alpha$-melanocyte stimulating hormone ( $\alpha$ MSH) neurons in the rat brain. Brain Res 220: 199-202, 1981.

5. Haglund, L., L. W. Swanson and C. Köhler. The projection of the supramammillary nucleus to the hippocampal formation: An immunohistochemical and anterograde transport study with the lectin PHA-L in the rat. $J$ Comp Neurol 229: 171-185, 1984.

6. Hatton, G. E. and W. E. Armstrong. Hypothalamic function in the behavioral and physiological control of body fluids. In: Behavioral Studies of the Hypothalamus. Handbook of the Hypothalamus, vol 3, part B, edited by P. J. Morgane and J. Panksepp. New York: Marcel Dekker, 1981, pp. I-105.

7. Jegou, S., M. C. Tonon, J. Guy, H. Vaudry and G. Pelletier. Biological and immunological characterization of $\alpha$-melanocyte stimulating hormone ( $\alpha-\mathrm{MSH})$ in two neuronal systems of the rat brain. Brain Res 260: 91-98, 1983.

8. Khachaturian, H., M. E. Lewis, S. Tsou and S. J. Watson. Beta-endorphin, $\alpha-\mathrm{MSH}, \mathrm{ACTH}$ and related peptides. In: Handbook of Chemical Neuroanatomy. Volume 4, Neuropeptides in the CNS, edited by T. Hokfelt and A. Bjorklund. Amsterdam: Elsevier, 1985, in press.

9. Khachaturian, H., K. Tsou and S. J. Watson. Beta-endorphin and $\alpha-\mathrm{MSH}$ in the rat brain: A comparative immunocytochemical analysis. Soc Neurosci Abstr 7: 93, 1981.

10. Kievet, J. and H. G. J. M. Kuypers. Basal forebrain and hypothalamic connections to the frontal and parietal cortex of the rhesus monkey. Science 187: 660-662, 1975.
11. Kobobun, K., T. L. O'Donohue, G. E. Handelmann, T. K. Sawyer, V. J. Hruby and M. E. Hadley. Behavioral effects of [4-Norleucine,7-Phenylalanine]- $\alpha$-melanocyte-stimulating hormone. Peptides 4: 721-724, 1983.

12. Köhler, C, , L. Haglund and L. W. Swanson. A diffuse $\alpha-$ MSH-immunoreactive projection to the hippocampus and spinal cord from individual neurons in the lateral hypothalamic area and zona incerta. J Comp Neurol 223: 501-514, 1984.

13. Köhler, C. and L. W. Swanson. Acetylcholinesterasecontaining cells in the lateral hypothalamic area are immunoreactive for $\alpha$-melanocyte stimulating hormone $(\alpha-\mathbf{M S H})$ and have cortical projections in the rat. Neurosci Lett 49: 39-43, 1984.

14. Nagy, J. I., L. A. LaBella, M. Buss and P. E. Daddona. Immunohistochemistry of adenosine deaminase: Implication for adenosine transmission. Science 224: 166-168, 1984.

15. Panula, P., H.-Y. Yang and E. Costa. Histamine-containing neurons in the rat hypothalamus. Proc Natl Acad Sci USA 81: 2572-2576, 1984 .

16. Pfaff, D. W. and B. S. McEwen. Actions of estrogens and progestins on nerve cells. Science 219: 808-814, 1983.

17. Powley, T. L., C. A. Opsahl, J. E. Cox and H. P. Weingarten. The role of the hypothalamus in energy homeostasis. In: Behavioral Studies of the Hypothalamus. Handbook of the Hypothalamus, vol 3, part A, edited by P. J. Morgane and J. Panksepp. New York: Marcel Dekker, 1980, pp. 211-298.

18. Rye, D. B., B. H. Wainer, M.-M. Mesulam, E. J. Mufson and C. B. Saper. Cortical projections from the basal forebrain: A study of cholinergic and non-cholinergic components employing combined retrograde tracing and immunohistochemical localization of choline acetyltransferase. Neuroscience 13: 627$643,1984$.

19. Saper, C. B. Organization of cerebral cortical afferent systems in the rat. II. Hypothalamocortical projections. J Comp Neurol 237: $21-46,1985$.

20. Satinoff, E. Neural organization and evolution of thermal regulation in mammals. Science 201: 16-22, 1978.

\section{FACING PAGE}

FIG. 8. A series of fluorescence photomicrographs to illustrate the appearance of retrogradely labeled, $\alpha$-MSH-lir neurons in the hypothalamus, in the same experiment shown in Fig. 7. Each pair of photomicrographs shows the same field under fluorescence illumination to demonstrate retrogradely transported dyes (panels A, C. E, G) and FITC fluorescence for $\alpha$-MSH-lir (paneis B, D. F, H). Panel A shows a group of six fast blue labeled neurons (arrows), in the lateral part of the dorsomedial nucleus; in panel B it can be seen that all six neurons are $\alpha$-MSH-lir. Scale for panels $A, B=100 \mu \mathrm{m}$. In panel $\mathrm{C}$, three fast blue (arrows) and one diamidino yellow (arrowhead) labeled neurons in LHAt can be seen to correspond to $\alpha$-MSH-lir perikarya in Fig. D. Similarly, zona incerta neurons retrogradely labeled with fast bilue (arrow) and diamidino yellow (arrowhead) dyes in panel $\mathrm{E}$ are shown to be $\alpha$-MSH-lir in panel F. In panel G, one LHAt neuron is shown which contained both retrograde labels (open arrow). Two other neurons, one labeled with fast blue (arrow) and one with diamidino yellow (arrowhead) are overlapping, giving the appearance of a double-labeled cell. In panel $\mathbf{H}$, it is clearer that these two neurons are in different focal planes; all three retrogradely labeled cells are $\alpha$-MSH-lir. Scale for panels $\mathrm{C}-\mathrm{H}=\mathbf{5 0} \mu \mathrm{m}$. 
21. Satinoff. E. Are there similarities between thermoregulation and sexual behavior? In: The Phvsolegical Me'hanisms of Motivaliom. edited by D. W. Pfaff. New York: Springer-Verlag. 1982. pp. $217-251$

22. Shiosaka, S., T. Shibasaki and M. Tohyoma. Bilateral $\alpha$-melanocyte stimulating hormonergic fiber system from zona incerta to cerebral cortex: a combined retrograde axonal transport and immunohistochemical study. Brin Res 309: 350-353. 1984.

23. Stevenson. J. A. F. Neural control of food and water intake. In: The Hypothalamus, edited by $W$. Haymaker, E. Anderson and W. J. H. Nauta. Springfield, IL: Chatles C. Thomas. 1979, pp. $524-62 !$.

24. van Wimersma Greidanus, T. B., B. Bohus. G. L. Kovacs, D. H. G. Versteeg. J. P. H. Burbach and D. De Wied. Sites of behavioral and neurochemical action of ACTH-like peptides and neurohypophyseal hormones. Neurusci Biobohav Rev 7 : $453-463,1983$,
25. Vincent. S. R.. T. Hokfelt, L. R. Skirboll and I. Y. Hu Hypo thalamic $\gamma$-aminobutyric acid neurons proied to the neocortex. Scichec 220: 1309-1311, 1983.

26. Watson. S. J. $\alpha-\mathrm{MSH}$ in brain $\beta$-endorphin neurons, and whor neurons as well. In: Endoughous and Elugenums Opiate A kemists and Antagemists, edited by R. 1. Way. Now York: Pergamon Press. 1980. pp. 127-130.

27. Watson, S. J, and H. Akil. The presence of two $\alpha$-MSH positive cell groups in rat hypothalamus, Eu , Phamotes/ 58: 101-103. 1979.

28. Watson, S. J. and $\mathrm{H}$. Akil. $\alpha-\mathrm{MSH}$ in ral brain: occurrence within and outside of $\beta$-endorphin neurons. Brain Res 182: $217-223,1980$.

29. Wayner, M. J., F. C. Barone and C.C. Loullis, The lateral hypothakmus and adjunctive behavior. In: Bethotoral Stalies of the Hypothalamus. Hondhosk of the Hypothalemus. vol 3. part B. edited by P. J. Morgane and J. Panksepp. New York: Marcel Dekker, 1981, pp. 107-145. 